[17] H. Kwakernaak, "Filtering for systems excited by Poisson white noise," in Lecture Notes in Economics and Mathematical Systems, A. Bensoussan and J. L. Lions Eds., vol. 107. New York: Springer-Verlag, 1975, pp. $468-493$.

[18] T. Björk, "Finite dimensional optimal filters for a class of Itô processes with jumping parameters," Stochastics, vol. 4, pp. 167-183, 1980.

[19] T. Başar and P. Bernard, $H \infty$-Optimal Control and Related Minimax Design Problems: A Dynamic Game Approach. Boston: Birkhäuser, 1991.

[20] L. J. Campo and Y. Bar-Shalom, "A new controller for discrete-time stochastic systems with Markovian Jump parameters," in Proc. 11th IFAC World Congr., Aug. 1990.

[21] H. A. P. Blom and M. H. C. Everdij, "Embedding adaptive JLQG into LQ Martingale with a completely observable stochastic control matrix," in Proc. 32nd IEEE Conf. Decision Contr., Dec. 1993, pp. 849-854.

[22] R. J. Elliott, Stochastic Calculus and Applications. New York: Springer-Verlag, 1982.

[23] R. W. Brockett and G. L. Blankenship, "A representation theorem for linear differential equations with Markovian coefficients," in Proc. Allerton Conf. Circuit Syst. Theory, 1977, pp. 671-679.

[24] M. H. C. Everdij and H. A. P. Blom, "Embedding adaptive JLQG into LQ Martingale control with a completely observable stochastic control matrix," National Aerospace Laboratory NLR, Report TP 95202. Amsterdam, 1995.

[25] W. H. Fleming and R. W. Rishel, Deterministic and Stochastic Optimal Control. Berlin: Springer-Verlag, 1975.

\section{On the Order of Simultaneously Stabilizing Compensators}

\author{
Onur Toker
}

Abstract-In this paper, simultaneous strong stabilization problem is considered, and it is shown that there is no upper bound for the minimal order of a simultaneously strongly stabilizing compensator, in terms of the plant orders. A similar problem was also considered in [11], where it was shown that such a bound does not exist for the strong stabilization problem of a single plant. But the examples given in [11] were forcing an approximate unstable pole-zero cancellation or forcing the distance between two distinct unstable zeros to go to zero. In this paper it is shown that: i) If approximate unstable pole-zero cancellation does not occur, and the distances between distinct unstable zeros are bounded below by a positive constant, then it is possible to find an upper bound for the minimal order of a strongly stabilizing compensator, and ii) for the simultaneous strong stabilization problem (even for the two plant case), such a bound cannot be found.

\section{INTRODUCTION}

It is known that a plant $P$ of order $n$ can be internally stabilized ${ }^{1}$ by a compensator $C$ of order, at most, $n-1$. As shown in [11], if the compensator is required to be stable, no such bound exists in terms of the plant order. The examples given in [11] are

$$
\begin{aligned}
& G_{\epsilon}(s)=\frac{(s-1)(s-2)}{s(s-2-\epsilon)(s-3)} \\
& H_{\epsilon}(s)=\frac{(s-(1+j \epsilon))(s-(1-j \epsilon))}{s^{2}(s-2)}
\end{aligned}
$$

Manuscript received July 28, 1994; revised March 31, 1995. This work was supported in part by the NSF under Grant MSS-920341 and by AFOSR under Grant F49620-93-1-0288.

The author is with the Eindhoven University of Technology, The Netherlands.

Publisher Item Identifier S 0018-9286(96)02109-5.

${ }^{1}$ Throughout this paper, only proper plants are considered and stability means internal stability [13]. and as $\epsilon \rightarrow 0^{+}$, the minimal order of a strongly stabilizing compensator goes to infinity [11]. It is clear that the first example forces an approximate unstable pole-zero cancellation, and the second one forces the distance between two distinct unstable zeros to go to zero.

In Section II, it will be shown that as long as approximate unstable pole-zero cancelation is not forced and the distances between distinct unstable zeros are not forced to go to zero, i.e., as long as we know a positive lower bound, $\delta_{0}$; for $\delta(P)=\max \left\{\delta_{1}(P), \delta_{2}(P)\right\}$ where

$$
\begin{aligned}
& \delta_{1}(P)=\frac{\min \left\{\left|z_{o}-z_{p}\right|: z_{o} \text { is a } \mathbb{C}_{+} \text {zero, } z_{p} \text { is a } \mathbb{C}_{+} \text {pole of } P\right\}}{\max \{|z|: z \text { is a } \mathbb{C} \text { pole or zero of } P\}} \\
& \delta_{2}(P)=\frac{\min \left\{\left|z_{1}-z_{2}\right|: z_{1}, z_{2} \text { are distinct } \mathbb{C}_{+}+\text {zeros of } P\right\}}{\max \{|z|: z \text { is a } \mathbb{C} \text { pole or zero of } P\}}
\end{aligned}
$$

if $P(s) \neq k / s^{n}$, otherwise $\delta_{1}(P)=\delta_{2}(P)=1$, and $\mathbb{C}_{+}$is the closed right-half plane; it is possible to find an upper bound on the minimal order of a strongly stabilizing compensator. Namely, there exists an upper bound $M\left(n, \delta_{0}\right)$ such that, if $P$ is strongly stabilizable, then $P$ is strongly stabilizable by a compensator of order, at most, $M\left(n, \delta_{0}\right)$.

In Section III, it will be shown that as $\beta \rightarrow\left(4 \pi^{2} / \Gamma^{4}(1 / 4)\right)^{+}$, the minimal order of a compensator which simultaneously strongly stabilizes

$$
\begin{aligned}
& P_{1, \beta}(s)=\frac{(s-1)^{2}}{(1+\beta)(s+1)\left(s-\frac{1-\beta}{1+\beta}\right)} \\
& P_{2, \beta}(s)=\frac{(s-1)^{2}}{(1-\beta)(s+1)\left(s-\frac{1+\beta}{1-\beta}\right)}
\end{aligned}
$$

goes to infinity. Note that $\delta\left(P_{1, \beta}\right)$ and $\delta\left(P_{2, \beta}\right)$ are bounded below by a positive number. The results of Section III are based on [3].

The simultaneous stabilization problem of $n$ plants is equivalent to the simultaneous strong stabilization problem of $n-1$ plants [13]. Stabilization by a stable compensator problem is also called the strong stabilization problem. For the one plant case, strong stabilizability is equivalent to the so-called parity interlacing property [17]. But the problem of stabilizing two plants with a stable compensator (equivalently the problem of simultaneously stabilizing three plants) seems to be more difficult. Several necessary conditions [5], [15] and sufficient conditions [16], [2], [8] are known as well as necessary and sufficient ones which involve untractable transcendental equations [6], [4]. Recently, Blondel and Gevers showed that the simultaneous stabilization problem of three plants (equivalently the simultaneous strong stabilization problem of two plants) is rationally undecidable [3]. This means that it is impossible to find a necessary and sufficient condition which involves only rational operations (i.e., addition, subtraction, multiplication, and division) on the coefficients of the plants, inequalities (i.e., $>, \geq,<, \leq$ ) and logical connectives (i.e., AND, OR, NOT). For example, the well-known Routh-Hurwitz test for the stability of a polynomial $p(s)$ involves only rational operations on the coefficients of $p(s)$, inequalities, and logical connectives, hence the stability of a polynomial $p(s)$ is rationally decidable. Similarly, the strong stabilization problem is rationally decidable, because parity interlacing property can be checked by using only rational operations [1]. The result presented in [3] shows that no such simple iff type of condition can be found for the simultaneous strong stabilization problem of two plants. Using these observations, in Section III it will be shown that the minimal compensator order may be unbounded for the simultaneous stabilization problem of three plants, equivalently for the simultaneous strong stabilization problem of two plants even if $\delta(P)^{\text {'s }}$ are bounded below by a positive number. 


\section{Strong Stabilization: One Plant Case}

In this section, it is shown that given the order $n$ of a plant $P$ and a positive lower bound $\delta_{o}$ for $\delta(P)$, it is possible to find an upper bound $M\left(n, \delta_{o}\right)$ for the minimal order of a strongly stabilizing compensator. Define $v(P)=$ minimal order of a compensator which strongly stabilizes $P(s)$. Without loss of generality, we may assume that $P(s) \neq k / s^{n}$. Because $k \neq 0, C(s)$ strongly stabilizes $1 / s^{n}$ iff $C(s) / k$ strongly stabilizes $k / s^{n}$, so $v\left(k / s^{n}\right)=v\left(1 / s^{n}\right)$ for all $k \neq 0$. Therefore, $\sup _{k \neq 0} v\left(k / s^{n}\right)=v\left(1 / s^{n}\right)<\infty$. Define

$$
\kappa=2 \max \{|z|: z \text { is a } \mathbb{C} \text { pole or zero of } P(s)\} \neq 0 \text {. }
$$

Since $C(s)$ strongly stabilizes $P(s)$ iff $C(\kappa s)$ strongly stabilizes $P_{1}(s):=P(\kappa s)$, we have $v(P(s))=v\left(P_{1}(s)\right)$. Note that, all $\mathbb{C}$ poles of $P_{1}(s)$ have absolute value $\leq 1 / 2$, and the distance between a $\mathbb{C}_{+}$pole and a $\mathbb{C}_{+}$zero of $P_{1}(s)$ as well as the distance between two distinct $\mathbb{C}_{+}$zeros of $P_{1}$ is greater than $\delta_{0} / 2$. Let $p_{1}(z):=P_{1}((1+z) /(1-z))$, then $c_{1}(z)$ strongly stabilizes $p_{1}(z)$ iff $C(s):=\left.c_{1}(z)\right|_{z=(s-1) /(s+1)}$ strongly stabilizes $P(s)$. Note that the orders of $P_{1}$ and $p_{1}$ are the same as the orders of $C_{1}$ and $c_{1}$. The distance between a $\overline{\mathbb{D}}$ pole and a $\overline{\mathbb{D}}$ zero of $p_{1}(z)$ as well as the distance between two distinct $\overline{\mathbb{D}}$ zeros of $p_{1}$ is greater than $\hat{\delta}_{o}:=\min \left\{1, \delta_{o} / 4\right\}$. Because $s_{1}, s_{2} \in \mathbb{C}_{+}$with $\left|s_{1}\right|,\left|s_{2}\right| \leq 1 / 2$ and $\left|s_{1}-s_{2}\right|>\delta_{0} / 2$

$$
\left|\frac{s_{1}-1}{s_{1}+1}-\frac{s_{2}-1}{s_{2}+1}\right|=\frac{2\left|s_{1}-s_{2}\right|}{\left|\left(s_{1}+1\right)\left(s_{2}+1\right)\right|}>\frac{\delta_{0}}{4} .
$$

Furthermore, the point $s=\infty$ in the s-plane is mapped to $z=1$ in the $z$-plane. If $s_{1} \in \mathbb{C}_{+}$with $\left|s_{1}\right| \leq 1 / 2$, then

$$
\left|\frac{s_{1}-1}{s_{1}+1}-1\right|=\frac{2}{\left|s_{1}+1\right|}>1
$$

hence the positive lower bound $\hat{\delta}_{0}:=\min \left\{1, \delta_{0} / 4\right\}$ is justified. Furthermore, poles and zeros of $p_{1}(z)$ have absolute value $\leq 4$, because if $s \in \mathbb{C}$ and $|s| \leq 1 / 2$, then $|(s-1) /(s+1)| \leq 4$ and $s=\infty$ is mapped to $z=1$ which is also $\leq 4$ in absolute value.

Let $p_{1}(z)=n_{1}(z) / d_{1}(z)$, where $n_{1}(z)$ and $d_{1}(z)$ are polynomials with no common root (hence coprime) with a leading coefficient of $d_{1}(z)$ equal to 1 . The distance between a $\overline{\mathbb{D}}$ root of $n_{1}(z)$ and a $\overline{\mathbb{D}}$ root of $d_{1}(z)$ as well as the distance between two distinct $\overline{\mathbb{D}}$ roots of $n_{1}(z)$ are greater than $\hat{\delta}_{0}$. To prove that the controller order remains bounded, we will use the unit construction procedure given in [13] and show that it is possible to find a bound on the order of the associated unit. This clearly implies that it is possible to find a bound on the controller order too, and a bound on the controller order in $z$-domain implies the existence of a similar bound on the controller order in the $s$-domain.

Let $n_{1}(z)=\left(z-z_{1}\right)^{r_{1}} \cdots\left(z-z_{t}\right)^{r_{t}}\left(z-z_{t+1}\right)^{r_{t+1}} \cdots\left(z-z_{n}\right)^{r_{n}}$ with $z_{1}, \cdots, z_{t} \in \overline{\mathbb{D}}$ and $z_{t+1}, \cdots, z_{n} \in \mathbb{C} \backslash \overline{\mathbb{D}}$. First, assume that we know $t$ and $r_{1}, \cdots, r_{t}$ but don't know the exact location of $z_{1}, \cdots, z_{t}$ in $\overline{\mathbb{D}}$. If it is possible to find a bound on the order of the associated unit with this much of information, then it is possible to find a bound which depends only on $n$ and $\delta_{0}$, because there are finitely many possibilities for $t$ and $r_{1}, \cdots, r_{t}$. In the remaining part of the section, it is assumed that $n$ and $\delta_{0}$ are known as well as $t$ and $r_{1}, \cdots, r_{t}$.

Let us look at more closely the unit construction procedure given in [13, Section 2.4] (interpolation in the disc algebra). Namely, we would like to construct a unit $f(z)$ such that $\left(f(z)-d_{1}(z)\right) / n_{1}(z)$ is stable. Once such a unit $f(z)$ is constructed, $c(z)=\left(f(z)-d_{1}(z)\right) / n_{1}(z)$ is a strongly stabilizing controller for $p_{1}(z)=n_{1}(z) / d_{1}(z)$. First, construct a polynomial $g(z)$ of order $r_{1}+\cdots+r_{t}$ such that

$$
\begin{gathered}
\left.\frac{d^{j}}{d z^{j}} e^{g(z)}\right|_{z=z_{i}}=\left.\frac{d^{j}}{d z^{j}} d_{1}(z)\right|_{z=z_{i}}, \quad i=1, \cdots, t, \\
j=0, \cdots, r_{i}-1 .
\end{gathered}
$$

Namely

$$
\begin{aligned}
g\left(z_{i}\right) & =\log \left(d_{1}\left(z_{i}\right)\right) \\
g^{\prime}\left(z_{i}\right) & =d_{1}^{\prime}\left(z_{i}\right) / d_{1}\left(z_{i}\right) \\
g^{\prime \prime}\left(z_{i}\right) & =\left(d_{1}^{\prime \prime}\left(z_{i}\right)-\left[d_{1}^{\prime}\left(z_{i}\right)\right]^{2}\right) / d_{1}\left(z_{i}\right) \\
\vdots & \vdots
\end{aligned}
$$

This gives a linear system of equations for the coefficients of $g(z)$. Since $d_{1}(z)$ is an $n$th order polynomial with leading coefficient equal to 1 and all of the roots of $d_{1}(z)$ have absolute value $\leq 4$, distance of a root of $d_{1}(z)$ to any of the $z_{i}$ 's is at least $\hat{\delta}_{o}$, and $z_{i}$ 's are in $\overline{\mathbb{D}}$, the right-hand sides of the linear system of equations given in (2) are bounded from above (in absolute value) by numbers which depend only on $n, \hat{\delta}_{o}, t, r_{1}, \cdots, r_{t}$ but are independent of the exact location of $z_{i}$ 's. Furthermore, the determinant of the left-hand side of (2) is bounded from below (in absolute value), and the entries of the adjoint matrix of the left-hand side of (2) are bounded from above (in absolute value) by numbers which depend only on $n, \hat{\delta}_{o}, t, r_{1}, \cdots, r_{t}$ but are independent of the exact location of $z_{i}$ 's. This is because all of the $z_{i}$ 's are in $\overline{\mathbb{D}}$ and the distance between distinct $z_{i}$ 's is at least $\hat{\delta}_{o}$. Therefore, it is possible to find upper bounds for the absolute values of the coefficients of $g(z)$ which depend only $n, \hat{\delta}_{o}, t, r_{1}, \cdots, r_{t}$ but are independent of the exact location of $z_{i}$ 's.

At this point, we know the existence of an upper bound for the absolute values of the coefficients of $g(z)$, independent of the exact location $z_{i}$ 's. Therefore $\exists M_{1}\left(n, \delta_{0}\right)$ such that for all $z \in \overline{\mathbb{D}}$

$$
\left|\frac{d^{j}}{d z^{j}} g(z)\right| \leq M_{1}\left(n, \delta_{o}\right), \quad j=0, \cdots, n-1
$$

therefore

$$
\left|\frac{d^{j}}{d z^{j}} g(z)^{k}\right| \leq k^{j} M_{1}\left(n, \delta_{0}\right)^{k}
$$

Since the infinite series

$$
\sum_{k=1}^{\infty} \frac{k^{j} M_{1}\left(n, \delta_{o}\right)^{k}}{k !}
$$

converges for all $j=0, \cdots, n-1$, there exists $N_{1}\left(n, \delta_{o}, \epsilon\right)$ which is independent of the exact locations of $z_{i}$ 's, such that for all $z \in \overline{\mathbb{D}}$

$$
\left|\frac{d^{j}}{d z^{j}} \sum_{k=N_{1}\left(n, \delta_{0}, \epsilon\right)}^{\infty} \frac{g(z)^{k}}{k !}\right| \leq \epsilon, \quad j=0, \cdots, n-1 .
$$

Now, by again Lagrange interpolation, there exists a polynomial $q(z)$ (which of course depends on the exact location of $z_{i}$ 's) such that

$$
\begin{gathered}
\left.\frac{d^{j}}{d z^{j}} q(z)\right|_{z=z_{i}}=\left.\frac{d^{j}}{d z^{j}} \sum_{\substack{k=N_{1}\left(n, \delta_{o}, \epsilon\right) \\
i}}^{\infty} \frac{g(z)^{k}}{k !}\right|_{z=z_{i}}, \\
j=0, \cdots, t, \\
j=0, r_{i}-1 .
\end{gathered}
$$

Since the distance between distinct $z_{i}$ 's are at least $\hat{\delta}_{o}$ and (4) holds, by a similar linear system of equations argument, it follows that the coefficients of $q(z)$ are bounded from above (in absolute value) by bounds which depend only on $n, \delta_{0}, t, r_{1}, \cdots, r_{t}, \epsilon$, and all of the upper bounds go to zero as $\epsilon$ goes to zero. Therefore, $\exists \Delta(n, \delta, \epsilon)$ such that for all $z \in \overline{\mathbb{D}}$

$$
|q(z)| \leq \Delta(n, \delta, \epsilon)
$$


and $\lim _{\epsilon \rightarrow 0} \Delta(n, \delta, \epsilon)=0$. Define

$$
f(z)=q(z)+\sum_{k=0}^{N_{1}(n, \delta, \epsilon)-1} \frac{g(z)^{k}}{k !} .
$$

By (4) and (5), $\left|e^{g(z)}-f(z)\right| \leq \epsilon+\Delta(n, \delta, \epsilon)$. Since $e^{g(z)}$ is a unit, if

$$
\sup _{z \in \overline{\mathbb{D}}}\left|e^{g(z)}-f(z)\right|<\left(\sup _{z \in \overline{\mathrm{D}}} \mid e^{-g(z)}\right)^{-1}
$$

then $f$ is a rational unit of order at most $n N_{1}(n, \delta, \epsilon)$ which interpolates $d_{1}(z)$ at $z_{i}$ 's up to $\left(r_{j}-1\right)^{\text {st }}$ derivates for $j=1, \cdots, t$. Note that by (3)

$$
e^{-M_{1}\left(n, \delta_{0}\right)} \leq\left(\sup _{z \in \overline{\mathbb{D}}}\left|e^{-g(z)}\right|\right)^{-1}
$$

Now choose $\epsilon=\epsilon(n, \delta)$ such that $\epsilon+\Delta(n, \delta, \epsilon)<e^{-M_{1}(n, \delta)}$ (This is possible because $\Delta(n, \delta, \epsilon)$ goes to zero as $\epsilon \rightarrow 0)$. Then, because of (7), (6) holds, so $f$ is a unit of order $\leq M_{f}(n, \delta):=n N_{1}(n, \delta, \epsilon(n, \delta))$ satisfying the appropriate interpolation conditions. With this construction, $c_{1}(z)=\left(f(z)-d_{1}(z)\right) / n_{1}(z)$ is a strongly stabilizing compensator for $p_{1}(z)$, and an upper bound on the order of the unit gives a similar upper bound $M(n, \delta)$ on order of the compensator.

The above results can be summarized in the following theorem.

Theorem 1: For a given $n$ and $\delta_{o}$, there exists a bound $M\left(n, \delta_{o}\right)$ such that a plant $P(s)$ of order $n$ and $\delta(P)>\delta_{0}$ is strongly stabilizable iff it is strongly stabilizable by a compensator of order at most $M\left(n, \delta_{0}\right)$.

Related with the above results, in [9] some bounds on the order of the units that satisfy a set of interpolation conditions are given. In that paper, a pair of $k$-tuples $\left(\left(z_{1}, \cdots, z_{k}\right),\left(w_{1}, \cdots, w_{k}\right)\right.$ ) (subject to $\left.\operatorname{Re}\left(z_{i}\right)>0\right)$ is called real if $z_{i}=\overline{z_{j}}$ implies $w_{i}=\overline{w_{j}}$, and is said to have the parity interlacing property if all the elements of the set $\left\{w_{i}: z_{i} \in \mathbb{R}\right\}$ have the same sign. Furthermore, the Nevanlinna-Pick matrix is defined as

$$
Q_{i, j}\left(\left(z_{1}, \cdots, z_{k}\right),\left(w_{1}, \cdots, w_{k}\right)\right)=\frac{w_{i}+\overline{w_{j}}}{z_{i}+\overline{z_{j}}} .
$$

Let

$$
\begin{gathered}
S_{m}=\left\{\left(v_{1}, \cdots, v_{k}\right): Q\left(\left(z_{1}, \cdots, z_{k}\right),\left(v_{1}, \cdots, v_{k}\right)\right)>0\right. \\
v_{i}^{m}=w_{i}, i=1, \cdots, k, \text { and }\left(\left(z_{1}, \cdots, z_{k}\right)\right. \\
\left.\left.\left(v_{1}, \cdots, v_{k}\right)\right) \text { is real }\right\}
\end{gathered}
$$

and $m_{0}$ be the smallest positive integer such that $S_{m_{0}}$ is not empty. Then, any unit $u$ satisfying $u\left(z_{i}\right)=w_{i}$, for $i=1, \cdots, k$, must be of order at least $m_{0}$, and there is an $m_{0} k t$ th degree unit satisfying the above interpolation conditions. Therefore, an upper bound on units can be obtained without actually computing the unit itself. The main disadvantage of this approach is that $\left(w_{1}^{1 / m}, \cdots, w_{k}^{1 / m}\right)$ is an $m^{k}$ valued $k$-tuple, and to check whether $S_{m}$ is empty or not, one has to consider $m^{k}$ possibilities.

\section{Strong Stabilization: Two Plant Case}

In this section, we show that as $\beta \rightarrow\left(4 \pi^{2} / \Gamma^{4}(1 / 4)\right)^{+}$, the minimal compensator order, which simultaneously stabilizes

$$
\begin{aligned}
& P_{0, \beta}(s)=0 \\
& P_{1, \beta}(s)=\frac{(s-1)^{2}}{(1+\beta)(s+1)\left(s-\frac{1-\beta}{1+\beta}\right)} \\
& P_{2, \beta}(s)=\frac{(s-1)^{2}}{(1-\beta)(s+1)\left(s-\frac{1+\beta}{1-\beta}\right)}
\end{aligned}
$$

equivalently the minimal compensator order which strongly stabilizes $P_{1, \beta}$ and $P_{2, \beta}$, goes to infinity. Note that orders of $P_{1, \beta}$ and $P_{2, \beta}$ remain the same as $\beta \rightarrow\left(4 \pi^{2} / \Gamma^{4}(1 / 4)\right)^{+}$, and neither approximate unstable pole-zero cancelation is forced, nor are the distances between distinct unstable zeros forced to go to zero, i.e.,

$$
\begin{aligned}
& \lim _{\beta \rightarrow\left(4 \pi^{2} / \Gamma^{4}(1 / 4)\right)^{+}} \delta\left(P_{1, \beta}\right)>0 \\
& \lim _{\beta \rightarrow\left(4 \pi^{2} / \Gamma^{4}(1 / 4)\right)^{+}} \delta\left(P_{2, \beta}\right)>0 .
\end{aligned}
$$

In [3], it is shown that if $|\beta|>4 \pi^{2} / \Gamma^{4}(1 / 4)$ ), then there exists a stable compensator which stabilizes $P_{1, \beta}$ and $P_{2, \beta}$, and if $|\beta|<4 \pi^{2} / \Gamma^{4}(1 / 4)$ ), then no such compensator exists. By the transcendence of $4 \pi^{2} / \Gamma^{4}(1 / 4)$, it follows that simultaneous stabilization problem by a stable compensator is rationally undecidable even for the pairs of plants $\left(P_{1, \beta}, P_{2, \beta}\right)$ [3].

Theorem 2: For $\beta>4 \pi^{2} / \Gamma^{4}(1 / 4)$, define $R(\beta)=$ minimal order of a compensator which strongly stabilizes $P_{1, \beta}$, and $P_{2, \beta}$. Then

$$
\lim _{\beta \rightarrow\left(4 \pi^{2} / \Gamma^{4}(1 / 4)\right)^{+}} R(\beta)=\infty .
$$

Proof: The proof will be given in two steps. First it will be shown that

$$
\sup _{\beta>4 \pi^{2} / \Gamma^{4}(1 / 4)} R(\beta)=\infty
$$

and then it will be shown that $R(\beta)$ is a nonincreasing function of $\beta$ for $\beta \in\left(\beta^{*}, \infty\right)$. These two results imply that

$$
\lim _{\beta \rightarrow\left(4 \pi^{2} / \Gamma^{4}(1 / 4)\right)^{+}} R(\beta)=\infty .
$$

First, note that if there exists a rational stable controller $C(s)$ stabilizing $P_{1, \beta}$ and $P_{2, \beta}$ for $|\beta|=4 \pi^{2} / \Gamma^{4}(1 / 4)$, then $C(s)$ should stabilize $P_{1, \beta \pm \epsilon}$ and $P_{2, \beta \pm \epsilon}$ for some $\epsilon>0$. This contradiction shows that for $|\beta|=4 \pi^{2} / \Gamma^{4}(1 / 4)$, there is no rational stable controller stabilizing $P_{1, \beta}$ and $P_{2, \beta}$. Now, define

$$
N=\sup _{\beta>4 \pi^{2} / \Gamma^{4}(1 / 4)} R(\beta) .
$$

To prove that $N$ is infinite, first assume that $N$ is finite, and show that this implies that the simultaneous stabilization problem of $P_{0, \beta}, P_{1, \beta}, P_{2, \beta}$, is rationally decidable (which contradicts with [3], and proves that $N=\infty$ ). If $N$ is finite, then to check simultaneous stabilizability by a stable compensator, it is enough to consider compensators $C$ with a state-space realization $\left(A, b, C^{T}, d\right)$ (possibly nonminimal), where $A$ is an $N$ by $N$ matrix, $b$ and $c$ are $N$ dimensional column vectors, and $d$ is a scalar. Let $\left(A_{i}, b_{i}, c_{i}^{T}, d_{i}\right)$ be controllable canonical form realizations of $P_{i, \beta}$ for $i=1,2$. Then the condition " $C$ strongly stabilizes $P_{1, \beta}$ and $P_{2, \beta}$ " is equivalent to the stability of $A, 1+d d_{i} \neq 0$ and the stability of the following two matrices:

$$
K_{i}:=\left[\begin{array}{cc}
A_{i}-\frac{1}{1+d d_{i}} b_{i} d c_{i}^{T} & -\frac{1}{1+d_{i}} b_{i} c^{T} \\
\frac{1}{1+d d_{i}} b c_{i}^{T} & A-\frac{1}{1+d d_{i}} b d_{i} c^{T}
\end{array}\right] .
$$

(These matrices correspond to the "A-matrix" of the closed-loop systems.) Note that stability of a matrix is rationally decidable in terms of the entries, i.e., first find the coefficients of the characteristic polynomial which will be polynomial expressions in terms of the entries, and then apply the Routh-Hurwitz criterion which will give 
a rational decision test in terms of coefficients of the characteristic polynomial hence in terms of the entries of the matrix. So, if we apply this rational decision test to the stability of $A, K_{1}$, and $K_{2}$, we will get a rational decision test for the simultaneous strong stabilizability of $\left(P_{1, \beta}, P_{2, \beta}\right)$ in terms of the entries of $A, b, c, d$ and $A_{i}, b_{i}, c_{i}, d_{i}, i=1,2$. At this point, we can use the Tarski's theorem [12], [7] or the Seidenberg elimination algorithm [7] to eliminate the entries of $A, b, c, d$ and obtain a new rational decision test in terms of only the entries of $A_{i}, b_{i}, c_{i}, d_{i}, i=1,2$. Therefore, this procedure will give a rational decision test for the simultaneous strong stabilizability of $\left(P_{1, \beta}, P_{2, \beta}\right)$ in terms of the coefficients of their numerators and denominators. But this contradicts the rational undecidability result of [3]. Therefore $N$ must be infinite, i.e.,

$$
\sup _{\beta>4 \pi^{2} / \Gamma^{4}(1 / 4)} R(\beta)=\infty .
$$

Remark: Tarski's theorem and the Seidenberg elimination algorithm are rather deep theoretical results. These results show that algebraic statements over real numbers [7] are decidable in finitely many steps. Both the Tarski's theorem and the Seidenberg elimination algorithm suffer from exponential growth and hence are not quite practical methods.

Now we know that $\sup _{\beta>4 \pi^{2} / \Gamma^{4}(1 / 4)} R(\beta)=\infty$, and to complete the proof of Theorem 2 , we prove that $R(\beta)$ is a nonincreasing function of $\beta$ for $\beta \in\left(\beta^{*}, \infty\right)$.

The compensator $C(s)$ simultaneously strongly stabilizes $P_{1, \beta}(s)$ and $P_{2, \beta}(s)$ iff the discrete-time compensator $c(z)=C((1+$ $z) /(1-z)$ ) simultaneously strongly stabilizes the discrete-time plants $p_{i, \beta}(z)=P_{i, \beta}((1+z) /(1-z))$ for $i=1,2$. By [3], we know that $c(z)$ simultaneously strongly stabilizes $p_{i, \beta}(z)$ for $i=1,2$ iff $c(z)$ is stable and

$$
\pm \beta+z+z^{2} c(z) \text { has no zeros on the closed unit disc. }
$$

This condition shows that if $c(z)$ is a simultaneously strongly stabilizing compensator for $p_{1, \beta}(z)$ and $p_{2, \beta}(z)$, then for $\Delta>0,(\beta / \beta+$ $\Delta) c((\beta / \beta+\Delta) z)$ is a simultaneously strongly stabilizing compensator for $p_{1, \beta+\Delta}(z)$ and $p_{2, \beta+\Delta}(z)$. Hence, $\tilde{C}(s)=(\beta / \beta+$ $\Delta)\left.c((\beta / \beta+\Delta) z)\right|_{z=(s-1) /(s+1)}$ is a simultaneously strongly stabilizing compensator for $P_{1, \beta+\Delta}(s)$ and $P_{2, \beta+\Delta}(s)$. Since the orders of $C(s)$ and $\tilde{C}(s)$ are the same, we obtain $R(\beta+\Delta) \leq R(\beta)$. Hence $R(\beta)$ is a nonincreasing function of $\beta$ for $\beta \in\left(\beta^{*}, \infty\right)$. This result together with $\sup _{\beta>4 \pi^{2} / \Gamma^{4}(1 / 4)} R(\beta)=\infty$ implies that

$$
\lim _{\beta \rightarrow\left(4 \pi^{2} / \Gamma^{4}(1 / 4)\right)^{+}} R(\beta)=\infty .
$$

The above results mean that for two arbitrary plants, $P_{1}(s)$ and $P_{2}(s)$, with given orders, $n_{1}$ and $n_{2}$, and a given positive lower bound $\delta_{0}$ for $\delta\left(P_{1}\right)$ and $\delta\left(P_{2}\right)$, it is impossible to find an upper bound $M\left(n_{1}, n_{2}, \delta_{0}\right)$ for the minimal order of a simultaneously strongly stabilizing compensator. Similarly, no bound exists for the simultaneous stabilization problem of three plants, even if we know the plants orders and a positive lower bound for $\delta\left(P_{i}\right)^{\text {'s. }}$

\section{CONCLUding Remarks}

In [11], it was shown by examples that, forcing an approximate unstable pole-zero cancelation or forcing the distance between two distinct unstable zeros to go to zero may force the minimal order of a strongly stabilizing compensator to go to infinity. In this paper, it is shown that as long as we know a positive lower bound $\delta_{0}$ for $\delta(P)$ and know the order $n$ of $P$, it is possible to find an upper bound $M\left(n, \delta_{0}\right)$ for the minimal order of a strongly stabilizing compensator. But such a bound cannot be found for the simultaneous strong stabilization problem of two plants and for the simultaneous stabilization problem of three plants.

\section{ACKNOWLEDGMENT}

The author would like thank H. Özbay, N. Gündeş, and K. A. Ünyeliog̃lu for their help and suggestions for improving this paper.

\section{REFERENCES}

[1] B. D. O. Anderson, "A note on the Youla-Bongiorno-Lu condition," Automatica, vol. 2, pp. 387-388, 1976.

[2] V. Blondel, G. Campion, and M. Gevers, "Avoidance and intersection in the complex plane as a tool for simultaneous stabilization," in Proc. 30th IEEE Conf. Decision Contr., Brighton, 1991, pp. 285-290.

[3] V. Blondel and M. Gevers, "Simultaneous stabilizability of three linear systems is rationally undecidable," Math. Contr., Signals Syst., pp. 135-145, 1993.

[4] V. Blondel, M. Gevers, R. Mortini, and R. Rupp, "Simultaneous stabilization of three or more plants: Conditions on the real axis do not suffice," SIAM J. Contr. Optimization, vol. 2, pp. 572-590, 1994.

[5] B. Ghosh, "Some new results on the simultaneous stabilizability of a family of single input single output systems," Syst. Contr. Lett., vol. 6, pp. $39-45,1985$.

[6] ___ "Transcendental and interpolation methods in simultaneous stabilization and simultaneous partial pole placement," SIAM J. Contr. Optimization, vol. 24, pp. 1091-1109, 1986.

17] N. Jacobson, Basic Algebra, vol. 1. New York: Freeman, 1985.

[8] H. Kwakernaak, "A condition for robust stabilizability," Syst. Contr. Lett., vol. 2, pp. 1005-1013, 1985.

[9] Y. Ohta, H. Maeda, and S. Kodama, "Unit interpolation in $\mathcal{H}$ : Bounds of norm and degree of interpolants," Syst. Contr. Lett. vol. 17, pp. 251-256, 1991.

[10] R. Saeks and J. Murray, "Fractional representation, algebraic geometry and the simultaneous stabilization problem," IEEE Trans. Automat. Contr., vol. AC-27, 895-903, 1982.

[11] M. C. Smith and K. P. Sondergeld, "On the order of stable compensators," Automatica, vol. 22, pp. 127-129, 1986.

[12] A. Tarski, "A decision method for elementary algebra and geometry," Berkeley note, 1951.

[13] M. Vidyasagar, Control System Synthesis: A Factorization Approach. Cambridge, MA: MIT Press, 1985.

[14] M. Vidyasagar and N. Viswanadham, "Algebraic design techniques for reliable stabilization," IEEE Trans. Automat. Contr., vol. AC-27, pp. 1085-1095, 1982.

[15] K. Wei, "The solution of a transcendental problem and its application in simultaneous stabilization problems," DLR Technical Report, Reference R38-91, 1991

[16] K. Wei and B. R. Barmish, "An iterative design procedure for simultaneous stabilization of MIMO systems," Automatica, vol. 24, pp. 643-652, 1988.

[17] D. C. Youla, J. J. Bongiorno, Jr., and C. N. Lu, "Single loop stabilization of linear multivariable dynamical plants," Automatica, vol. 10, pp 159-173, 1974. 